\title{
Extreme sensitivity of reservoir computing to small network disruptions
}

\author{
Philippe Vincent-Lamarre ${ }^{1 *}$, Guillaume Lajoie ${ }^{2,3}$, Jean-Philippe Thivierge ${ }^{1}$ \\ From 24th Annual Computational Neuroscience Meeting: CNS*2015 \\ Prague, Czech Republic. 18-23 July 2015
}

Recent computational models based on reservoir computing $(\mathrm{RC})$ are gaining attention as plausible theories of cortical information processing. In these models, the activity of a recurrently connected population of neurons is sent to one or many read-out units through a linear transformation. These models can operate in a chaotic regime which has been proposed as a possible mechanism underlying sustained irregular activity observed in
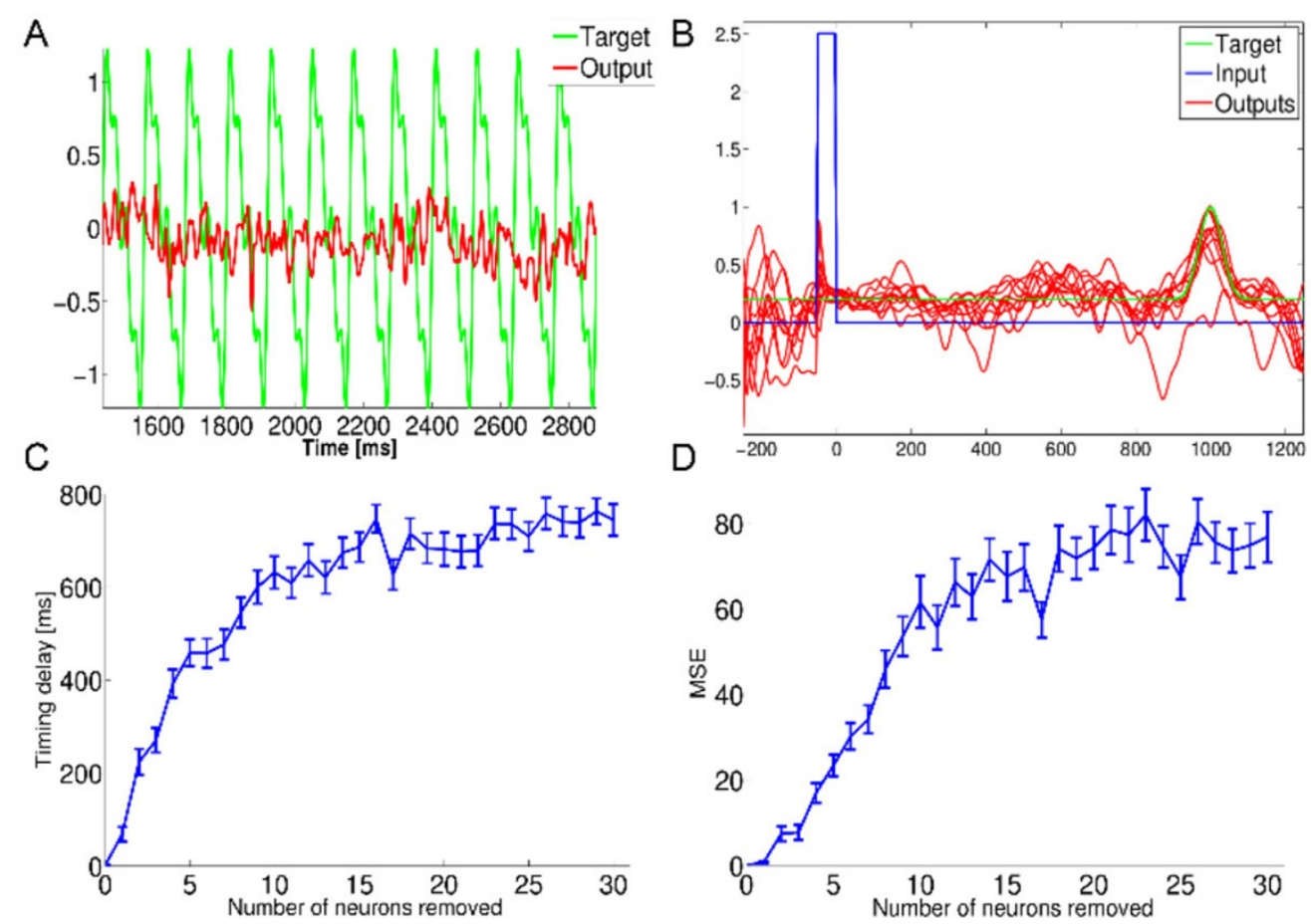

Figure 1 Performance of damaged reservoirs of 1,000 neurons with FORCE and innate learning algorithms. A. Target signal (green, perfectly replicated with the originally trained network) and the trace of the same network after the removal of one neuron in its reservoir. B. Ten trials (red) with different initial conditions of a damaged network ( $\mathrm{N}-2$ neurons) that is trained to peak at 1,000 ms (green) using innate learning. C. Average lag between the target and the output timing (100 trials per condition) as a function of the number of removed neurons. D. Mean squared error as a function of the number of removed neurons.

\footnotetext{
* Correspondence: pvinc058@uottawa.ca

${ }^{1}$ School of Psychology and Center for Neural Dynamics, University of Ottawa,

Ottawa, Ontario K1N 6N5, Canada

Full list of author information is available at the end of the article
}

(c) 2015 Vincent-Lamarre et al. This is an Open Access article distributed under the terms of the Creative Commons Attribution License (http://creativecommons.org/licenses/by/4.0), which permits unrestricted use, distribution, and reproduction in any medium, provided the original work is properly cited. The Creative Commons Public Domain Dedication waiver (http://creativecommons.org/ publicdomain/zero/1.0/) applies to the data made available in this article, unless otherwise stated. 
cortical areas [1,2]. Furthermore, models based on RC replicate the neural dynamics involved in decision making [3], interval timing [2], and motor control [1]. However, one biological constraint that has been overlooked in these models is their resistance to small connectivity perturbations such as failures in synaptic transmission, a phenomenon that occurs frequently in healthy circuits without causing any drastic functional changes. Here, we show that different implementations of RC display very little resistance to small synaptic disruptions and discuss the implications of such fragility for RC mechanisms that may be present in neural coding. With the FORCE [1] procedure, networks lost their ability to replicate a jagged sinusoidal signal after a single neuron was removed from the reservoir (Figure 1A). Networks with innate training [2] showed a similar effect on a timing task (Figure 1B). The lag in the timing and the noise in the output both increased monotonically as further neurons were removed (Figure 1C,D); networks reached random performance after $\sim 1.5 \%$ of neurons were eliminated. After the suppression of a single neuron, the spectrum of the weight matrix was greatly disturbed and repeated trials displayed unreliable trajectories, as assessed with principal components analysis. When individual synapses were removed instead of neurons, networks reached random performance after $\sim 0.5 \%$ of synapses from the reservoir were eliminated. While living neuronal circuits can withstand small synaptic disruptions without compromising task performance, our results suggest that such disruptions have a catastrophic impact on the behaviour of RC models. Retraining the read-out unit seems to be futile as it results as a completely new solution post retraining instead of a finer restructuration. These results cast doubt on the validity of a large class of models that claim to capture the neuronal mechanisms of cognitive and behavioral tasks.

\section{Acknowledgements}

This research was funded by grants to J.P.T. from NSERC Discovery and CIHR operating funds.

\section{Authors' details}

'School of Psychology and Center for Neural Dynamics, University of Ottawa, Ottawa, Ontario K1N 6N5, Canada. ${ }^{2}$ UW Institute for Neuroengineering, University of Washington, Seattle, WA, US. ${ }^{3}$ Max Planck Institute (DS) and Bernstein Center for Computational Neuroscience, Göttingen, Germany.

Published: 18 December 2015

\section{References}

1. Sussillo D, Abbott LF: Generating Coherent Patterns of Activity from Chaotic Neural Networks. Neuron 2009, 63:544-557.

2. Laje R, Buonomano DV: Robust timing and motor patterns by taming chaos in recurrent neural networks. Nat Neurosci 2013, 16:925-933.

3. Barak O, Sussillo D, Romo R, Tsodyks M, Abbott LF: From fixed points to chaos: Three models of delayed discrimination. Progress in Neurobiology 2013, 103:214-222
doi:10.1186/1471-2202-16-S1-P256

Cite this article as: Vincent-Lamarre et al:: Extreme sensitivity of reservoir computing to small network disruptions. BMC Neuroscience 2015 16(Suppl 1):P256.

\section{Submit your next manuscript to BioMed Central} and take full advantage of:

- Convenient online submission

- Thorough peer review

- No space constraints or color figure charges

- Immediate publication on acceptance

- Inclusion in PubMed, CAS, Scopus and Google Scholar

- Research which is freely available for redistribution

Submit your manuscript at www.biomedcentral.com/submit 